\title{
ERRATUM
}

\section{Erratum to: Genetic Research and Aboriginal and Torres Strait Islander Australians}

\author{
Emma Kowal • Glenn Pearson • Lobna Rouhani • \\ Chris S. Peacock • Sarra E. Jamieson • \\ Jenefer M. Blackwell
}

Received: 19 April 2011 / Accepted: 27 August 2012 /Published online: 9 July 2014

(C) Springer Science+Business Media Dordrecht 2014

\section{Erratum to: Bioethical Inquiry \\ DOI 10.1007/s11673-012-9391-x}

Lobna Rouhani, University of Melbourne, is a co-author of the article "Genetic Research and Aboriginal and Torres Strait Islander Australians" (2012, 419-432) that was published in the Journal of Bioethical Inquiry's 9(4) symposium "Cases and Culture." Her name was omitted from the publication and she should be credited as the third author of this article.

The online version of the original article can be found at http://dx.doi. org/10.1007/s11673-012-9391-x.

E. Kowal $(\bowtie) \cdot$ L. Rouhani

School of Social and Political Sciences,

University of Melbourne,

Melbourne, Victoria 3010, Australia

e-mail: e.kowal@unimelb.edu.au

G. Pearson · S. E. Jamieson · J. M. Blackwell

Telethon Institute for Child Health Research,

P.O. Box 855, West Perth, Western Australia 6872, Australia

C. S. Peacock

University of Western Australia,

35 Stirling Highway Crawley,

Perth, Western Australia 6009, Australia 